\title{
Phytobiome and Transcriptional Adaptation of Populus deltoides to Acute Progressive Drought and Cyclic Drought
}

\author{
Benjamin J. Garcia, ${ }^{1}$ Jessy L. Labbé, ${ }^{1}$ Piet Jones, ${ }^{1,2}$ Paul E. Abraham, ${ }^{3}$ lan Hodge, ${ }^{1,4}$ Sharlee Climer, ${ }^{5}$ \\ Sara Jawdy, ${ }^{1}$ Lee Gunter, ${ }^{1}$ Gerald A. Tuskan, ${ }^{1}$ Xiaohan Yang, ${ }^{1}$ Timothy J. Tschaplinski, ${ }^{1}$ and \\ Daniel A. Jacobson ${ }^{1,2, \dagger}$
}

${ }^{1}$ Center for Bioenergy Innovation, Oak Ridge National Laboratory, Oak Ridge, TN 37831; ${ }^{2}$ The Bredesen Center for Interdisciplinary Research and Graduate Education, University of Tennessee, Knoxville 37996 ; ${ }^{3} \mathrm{Chemical} \mathrm{Sciences}$ Division, Oak Ridge National Laboratory, Oak Ridge, TN 37831; ${ }^{4}$ Department of Computer Science, Stanford University, Stanford, CA 94305; and ${ }^{5}$ Department of Mathematics and Computer Science, University of Missouri, St. Louis 63121

Accepted for publication 29 November 2018.

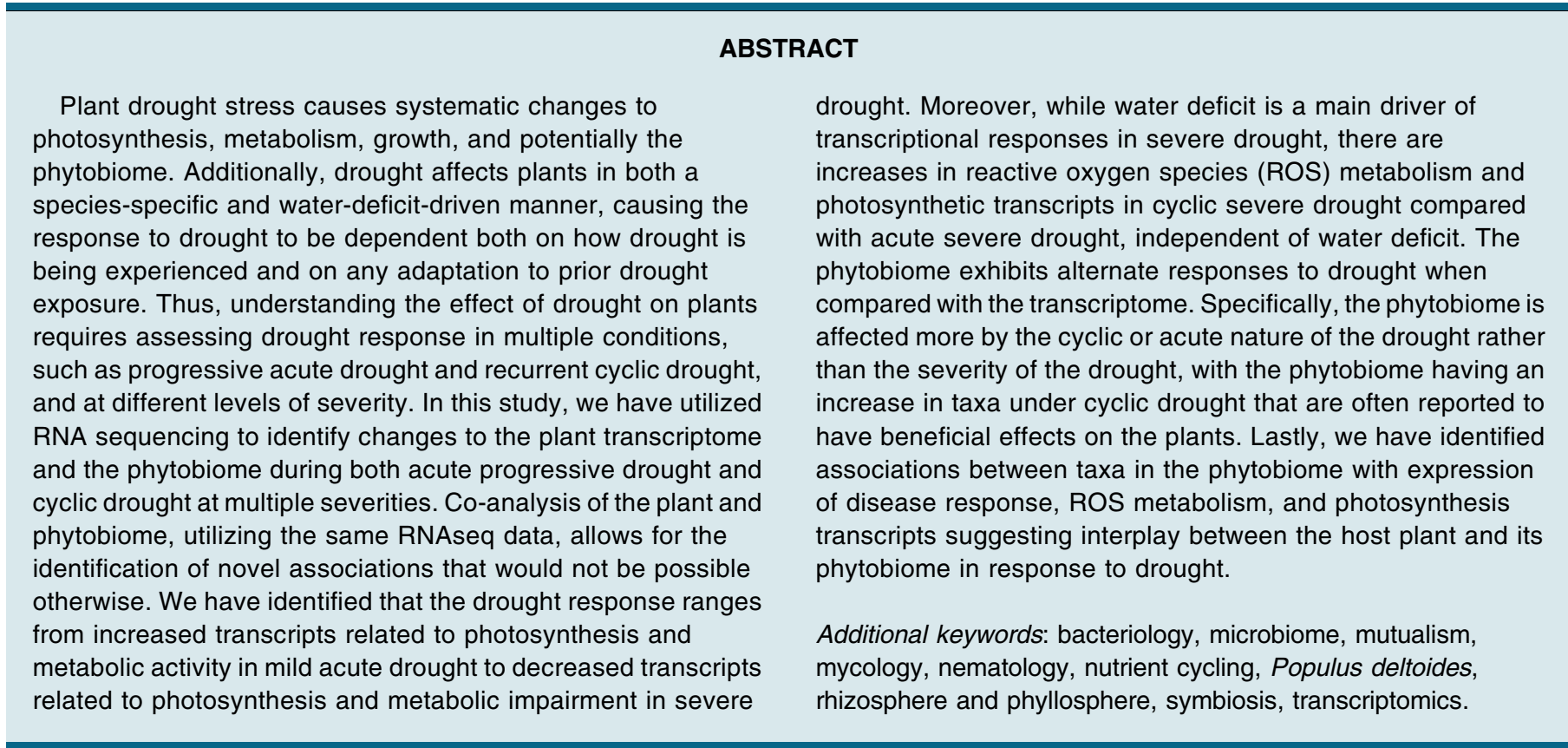

\section{${ }^{\dagger}$ Corresponding author: D. Jacobson; E-mail: jacobsonda@ornl.gov}

Funding: This research used resources of the Oak Ridge Leadership Computing Facility at the Oak Ridge National Laboratory, which is supported by the Office of Science of the U.S. Department of Energy under Contract No. DE-AC0500OR22725. This research was also supported by the Plant-Microbe Interfaces Scientific Focus Area in the Genomic Science Program, the Office of Biological and Environmental Research (BER) in the U.S. Department of Energy Office of Science, and by the Department of Energy, Laboratory Directed Research and Development funding (Project ID 8321), at the Oak Ridge National Laboratory. This work was also supported by the Center for Bioenergy Innovation, a U.S. Department of Energy Bioenergy Research Center supported by the Office of Biological and Environmental Research in the DOE Office of Science. Oak Ridge National Laboratory is managed by UT-Battelle, LLC, for the U.S. DOE under contract DE-AC05-00OR22725.
*The $\boldsymbol{e}$-Xtra logo stands for "electronic extra" and indicates that 16 supplementary tables, eight supplementary figures, and one supplementary file are published online.

This manuscript has been authored by UT-Battelle, LLC under Contract No. DEAC05-00OR22725 with the U.S. Department of Energy. The United States Government retains and the publisher, by accepting the article for publication, acknowledges that the United States Government retains a non-exclusive, paid-up, irrevocable, world-wide license to publish or reproduce the published form of this manuscript, or allow others to do so, for United States Government purposes. The Department of Energy will provide public access to these results of federally sponsored research in accordance with the DOE Public Access Plan (http://www. energy.gov/downloads/doe-public-access-plan). 
Drought plays a key role in plant development, limiting growth and metabolic activity, in addition to causing both episodic and climate-induced mortality (Allen et al. 2010). In the woody plant, Populus tremuloides, climate-driven drought mortality is the result of hydraulic impairment (Anderegg et al. 2012). However, response to drought is (i) often species-specific (Farooq et al. 2009), (ii) directly related to the intensity of the drought (Barghini et al. 2015; Farooq et al. 2009; Flexas et al. 2006), and (iii) directly related to genotype and time of day, (Hamanishi et al. 2015). Therefore, studies involving (i) alternate species, (ii) varying levels of water deficits, and (iii) differences in drought stress duration are required in order to obtain a holistic understanding of the effect of drought on plants. In response to drought, plants alter their metabolic activity, often decreasing photosynthesis (Flexas et al. 2006; Zandalinas et al. 2017) and glycolytic metabolism (O'Leary et al. 2011; Pan et al. 2016; Plaxton 1996), with more severe drought resulting in greater metabolic impairment (Flexas et al. 2006) and programmed cell death (Petrov et al. 2015).

In addition to the effects drought can have on plant metabolism, drought also has a significant impact on the phytobiome. Rhizobacteria and mycorrhizal fungi can have plant growth promoting effects under drought conditions through nitrogen fixation (Vurukonda et al. 2016) and increasing the availability of phosphorus (Zhang et al. 2017) and potassium (Deepika and Kothamasi 2014). Beneficial drought-related organisms appear to be selected for by acute water stress (Rolli et al. 2015), while plant growth promoting organisms may be recruited from soil in arid conditions under prolonged drought (Marasco et al. 2012), suggesting acute drought affects the plant and phytobiome differently than cyclic and seasonal droughts. Beneficial effects of the phytobiome include providing nutrients to the plant (Deepika and Kothamasi 2014; Vurukonda et al. 2016; Zhang et al. 2017), altering functional behavior of plant structures to better tolerate stress, such as increasing stomatal conductance (Marasco et al. 2012), and decreasing oxidative stress damage (Campos et al. 2015). Conversely, the phytobiome can also negatively impact plant growth during stress; for example, via disease occurrence. Disease susceptibility could be the result of a decreased oxidative burst (Bidzinski et al. 2016) that provides a benefit to plant survivability during drought and is promoted by drought resistance-promoting fungi (Campos et al. 2015). In addition to increased disease susceptibility under drought, combined moderate drought stress and pathogen stress can result in a higher induction of both responses to pathogens and drought resulting in less disease (Pandey et al. 2015); however, the response is both pathogen specific and drought severity specific (Sinha et al. 2016).

Traditional identification of microbiome abundance often relies on marker-based operational taxonomic units (OTU), such as $16 \mathrm{~S}$ variable region rRNA. Marker sequencing is only able to identify organisms in databases with the markers of interest, is biased toward the efficiency/precision of the given markers, is not easily scalable to identify all organisms across all kingdoms of life, and is not often practical to compare abundances across different markers. However, RNAseq offers the opportunity to identify both the host transcriptome and the high abundance organisms using wholegenome sequences of all available sequenced organisms. One drawback of the RNAseq approach is the large differences in size between markers and whole genomes (both sequence length and database size), where traditional methodologies used for marker taxa quantification may not be efficient or well suited for taxa identification. MetaPhlAn2 (Truong et al. 2015), a commonly used taxa assignment program, deals with the large increase in data by subselecting markers for bacteria, viruses, eukaryotes, etc., thus reducing the search space. A drawback to subselection is that in low abundance data, especially in the case where host data makes up the vast majority of reads, sensitivity and specificity is often decreased (Wood and Salzberg 2014). To overcome the loss in accuracy, kmer-based approaches, such as Kraken (Wood and Salzberg 2014), allow for fast and accurate mapping of reads to a given taxa by allowing submatching in a read (Wood and Salzberg 2014). Kmer approaches break up genomes into shorter sequences of $\mathrm{k}$ length. All possible kmers are created for a given genome, sequenced reads are then compared with the database of all possible kmers, and a taxa assignment is made based upon the most frequent occurrence of exact kmers matches for a given taxa. Kmers have the benefit of being much faster than alignments. One disadvantage is that kmer approaches often require a large amount of memory and computational power. Some methods, such as centrifuge (Kim et al. 2016), use an FM-index that can match different sequence lengths with much smaller databases, at the cost of some speed and precision due to combining some sequences with $99 \%$ similarity rather than exact matching.

In this study, we integrate traditional RNASeq analysis with kmer-based approaches to better understand the transcriptional and phytobiome changes that occur in $P$. deltoides mature leaves during both acute progressive drought and cyclic drought. During this experiment, plants were deprived of water until differences in water potentials (ranging from mild drought to severe drought) were observed. Additionally, classification of the non- $P$. deltoides transcripts using a kmer approach (Wood and Salzberg 2014) with whole genome sequences allows for the understanding of which organisms may be growing on the leaves, how they are impacted by differences in drought conditions, and what implications statistically significant differential changes may have on differences in the plant transcriptomic response. For this study, we used a modified version of Kraken (ParaKraken) to assign RNAseq data to taxa, also allowing for comparison of the phytobiome to the host plant transcriptome. Acute drought transcriptional monitoring allows for a better understanding of the initial responses to drought in both the plant and the phytobiome; whereas cyclic drought transcriptional monitoring suggests how both the plant and the phytobiome may adapt to drought conditions. To elucidate potential differences between acute and cyclic drought for hypothesis generation, we also performed a trend-based analysis on more severe drought conditions, highlighting changes in functional categories and phytobiome composition to identify categorical shifts between the two experiments.

\section{MATERIALS AND METHODS}

Plant materials and culture conditions. $P$. deltoides WV94 plants were initially grown in tissue culture and then transferred to leach tubes containing peat, vermiculite, and perlite in a 2:2:1 ratio. Osmocote (3.17 ml/liter), bone meal (1.06 ml/liter), gypsum (1.06 $\mathrm{ml} /$ liter $)$, and dolomite $(0.53 \mathrm{ml} / \mathrm{liter})$ were added to the leach tubes, and the plantlets were grown in mist beds for 2 weeks. The plants were then transferred to greenhouses and grown at $19^{\circ} \mathrm{C}$ with natural light through Excelite panels. Fertilizer (300 ppm of Peter's 20-20-20 NPK) was added to the plants every month. Plants were then exposed to two different drought experiments: acute drought and cyclic drought (Fig. 1).

Acute drought experiment. For the acute drought experiment, nine $P$. deltoides WV94 6-month-old, 15-cm cuttings were used for transcriptomic sampling. Prior to leaf sampling, these cuttings were grown for an additional 6 months in well-watered conditions $(-0.1$ $\mathrm{MPa}$ ). Plants averaged $88 \mathrm{~cm}$ in height and $0.31 \mathrm{~cm}$ in diameter. At the start of the acute drought experiment, watering was stopped until signs of leaf wilting were observed. Predawn water potentials 
were measured using a Scholander pressure cylinder with average and standard error from the mean (sem) potentials shown in Table 1. A leaf that was 10 below the apex and had a Leaf Plastochron Index (LPI) of 9 to 12 was sampled on days $0,5,7$, and 8 posttreatment. Water potentials were recorded on an adjacent leaf to the leaf sampled for RNAseq. Briefly, the leaf was quickly sampled from the plant and its petiole trimmed with a razor blade to provide a smooth surface for observation. The petiole was placed in a cork borer to guide it through a pierced rubber stopper and the leaf with the stopper placed into the pressure cylinder with only the petiole visible outside the chamber, which was then locked shut. The cylinder was then slowly pressurized with nitrogen gas and the balance pressure at which the water first emerged from the cut petiole surface was the leaf water potential (MPa). Randomly designated plants 1 to 3 were sampled on day 0 , plants 4 to 6 were sampled on day 7 , and plants 7 to 9 were sampled on day 5 and 8 , resulting in three biological replicates for each time point.

Cyclic drought experiment. For the cyclic drought experiment, eight $P$. deltoides WV94 6-month-old, 15-cm cuttings were grown, with four plants used for controls and four plants used for drought. Similar to the acute drought experiment, these plants were grown for an additional 6 months in well-watered conditions prior to applying the experimental treatment. Plants in the cyclic drought experiment were of similar stature to those in the acute drought experiment. The four controls were well watered for the entire experiment $(-0.1 \mathrm{MPa})$, while the four drought plants underwent four drought and three rewatering cycles. Drought plants were rewatered once the predawn water potential of -0.8 was reached, resulting in plants being rewatered on days 6, 12, and 20. Cyclic drought plant leaves with an LPI of 9 to $12(\sim 10$ leaves below the apex) were sampled once the predawn water potential of $-0.5 \mathrm{MPa}$ (mild) was reached (9 days after the last rewatering), with the same plants sampled under severe drought conditions a day later. Cyclic control plants were sampled at the same time as the mild plants. Average water potentials for all three samples, with four biological replicates each, with water potentials recorded on a neighboring leaf to the leaf sampled for RNAseq (Table 1). Both acute and cyclic drought leaves were processed in the same manner described below.

RNA sequencing and alignment. Mature leaves were flash frozen in liquid nitrogen and pulverized with a mortar and pestle. Total RNA was then extracted from each replicate for each treatment using Spectrum Plant Total RNA isolation kit (Sigma) according to their standard protocol. On-column DNase treatment was included during RNA isolation for removing gDNA contamination. Total RNA quantity and quality were assessed using a NanoDrop spectrophotometer (Thermo Scientific), with $6 \mu \mathrm{g}$ of total RNA for each sample. For the acute experiment, day $0,5,7$, and 8 drought samples were used for further analysis, while for the cyclic experiment, control, mild, and severe drought samples were used for further analysis (Supplementary Table S1). RNA was sequenced using stranded, paired-end reads with a sequence length of $151 \mathrm{bp}$ on a HiSEQ-2500 Rapid V2 sequencer at the DOE Joint Genome Institute. Trimming was initially performed on FASTQ files using Skewer (Jiang et al. 2014) with a minimum three-primeend quality score of 20 and minimum length of $100 \mathrm{bp}$. A Star index (Dobin et al. 2013) was created using the $P$. deltoides v2.1 genome (https://phytozome.jgi.doe.gov/pz/portal.html), and the trimmed reads were aligned to the index using Star (Dobin et al. 2013) (Supplementary Table S2). Genes with expression in less than $75 \%$ of the samples across treatments were removed, resulting in the removal of 17,342 genes (38.6\% of total genes). The resulting aligned read counts were transformed using voom mean-variance stabilization (Law et al. 2014) with the resulting counts normalized using TMM (Robinson and Oshlack 2010) to account for library biases (Supplementary Table S3).

Differential expression and gene ontology (GO) enrichment. Normalized counts were analyzed with fcros (Dembélé and Kastner 2014) for differential expression between each sample group with a $P$ value cutoff of 0.05 and an f-score of 0.9 (Supplementary Table S4 and S5). Enrichment of GO terms was performed on the list of significantly differentially expressed genes using a modified Fisher's exact test (Garcia et al. 2015; Hosack et al. 2003) using Benjamini-Hochberg multiple-testing correction (Benjamini and Hochberg 1995) with a false discovery rate of 0.05 (Supplementary Table S6 and S7). Sample groups underwent hierarchical clustering using a Pearson correlation coefficient on $\log _{2}$-fold change values with complete agglomeration using the R package pheatmap (Kolde 2012). Functional annotations were computed for the primary proteome using Mercator (Lohse et al. 2014) to generate MapMan annotations (Thimm et al. 2004), allowing for better identification of plantrelated terms. MapMan annotations associated with each transcript were then used to identify changes in plant-specific functions between each of the conditions (Supplementary Table S8 and S9).

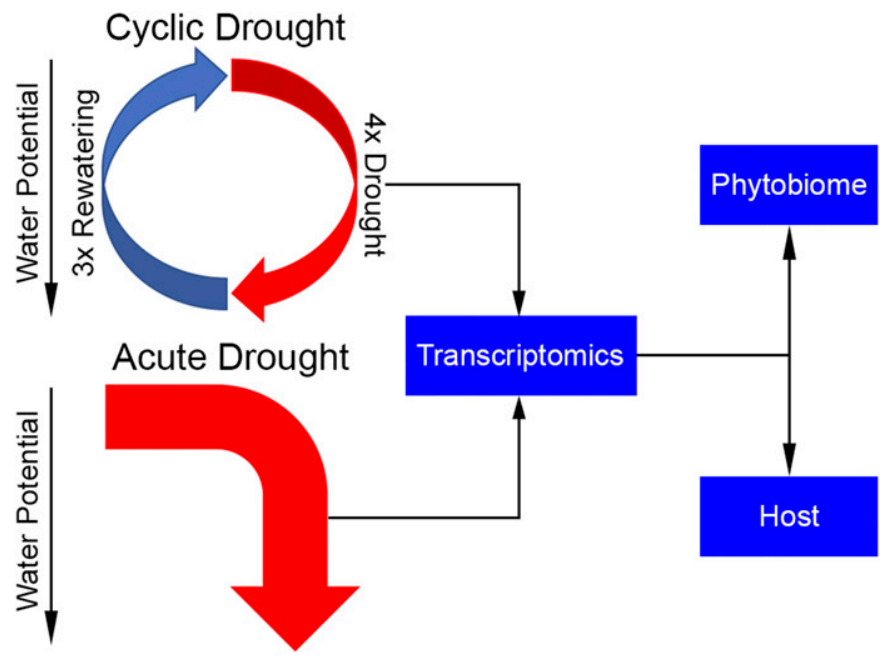

Fig. 1. Drought experimental design. Plants were exposed to two different drought conditions: acute drought (one drought exposure) and cyclic drought (four drought and three rewatering cycles). RNAseq was performed on fully expanded mature leaves ( $\sim 10$ leaves from the apex $)$ in both drought and control samples. Both the host transcriptome and phytobiome taxa were profiled and analyzed for differential expression/ abundance and pairwise associations.

TABLE 1

Water potentials for each sample group at the time of leaf sampling ${ }^{\text {a }}$

\begin{tabular}{lcc}
\hline Class & Average $(\mathrm{MPa})$ & sem $(\mathrm{MPa})$ \\
\hline Acute day 0 & -0.17 & 0.00 \\
\hline Acute day 5 & -0.44 & 0.08 \\
\hline Acute day 7 & -0.63 & 0.18 \\
\hline Acute day 8 & -1.85 & 0.32 \\
\hline Cyclic control & -0.11 & 0.02 \\
\hline Cyclic mild & -0.50 & 0.04 \\
\hline Cyclic severe & -1.26 & 0.12 \\
\hline
\end{tabular}

a Water potential was measured using a Scholander pressure cylinder on a neighboring mature leaf to transcriptome profiled leaf. sem, standard error from the mean. 
Phytobiome analysis and Kraken parallelization. Unmapped reads from the Star output were run through a modified and parallelized version of Kraken (Wood and Salzberg 2014). As it is impractical to create and run an entire Kraken database using whole genomes for all sequenced species and their resulting kmers, we developed a pipeline called ParaKraken. ParaKraken splits each of the whole-genome sequences into multiple databases, runs all samples against all databases in parallel, and then resolves each individual sample-database read pairs to generate taxonomic assignments (Supplementary Fig. S1). The resolution step uses the same lowest common ancestor method that Kraken uses for its taxa assignments. Additionally, the parallel modification is scalable to any number of whole genomes and any number of databases. Multiple database results from a single sample can be resolved in groups or sequentially, allowing for additional databases to be run and resolved without having to rerun the entire resolution step on all database results.

The modified Kraken pipeline was run on 169 databases containing whole genomes from $\sim 33 \mathrm{k}$ bacteria, 571 fungi, 734 archaea, 25 nematodes, 2 aphids, 7,103 viruses, and $P$. deltoides WV94 to quantify the phytobiome (Supplementary Table S10). Whole genomes were downloaded from NCBI RefSeq (O'Leary et al. 2016) in August 2016, with fungal whole genomes downloaded from JGI-IMG (Markowitz et al. 2012) in October 2016. Classified taxa in each sample represent the closest taxa in the databases of whole genome sequences. For genera with thousands of sequenced species, such as Pseudomonas, species/genus level resolution will be much higher than what would be found in a phyla with only 25 sequenced species (such as Nematoda, which will have a more apparent bias toward human pathogens).

A median scaling was utilized for each sample, where each sample total count was scaled by the median of all sample total counts. Rare taxa (taxa occurring in less than $75 \%$ of either sample group) were then removed from further analysis (Supplementary Table S11). fcros (Dembélé and Kastner 2014) was used to identify differential abundance between all sample groups using a $P$ value cutoff of 0.05 , an f-score of 0.9 , and an absolute $\log _{2}$-fold change greater than 1 (Supplementary Table S12 and S13). Scaled counts were then further standardized within a taxon, dividing all sample counts by the maximum sample count for a given taxon. Analysis of relationships between taxa was performed on the taxa scaled counts using DUO (https://github.com/climers/duo). DUO first identifies high (up) and low (down) abundance thresholds using 25th and 75th

\section{TABLE 2}

Total number of reads per sample and the number of reads mapping to the Populus deltoides transcriptome and to the phytobiome $^{\mathrm{a}}$

\begin{tabular}{llcc}
\hline Sample & All reads & $\begin{array}{c}P \text {. deltoides } \\
\text { transcriptome }\end{array}$ & Phytobiome \\
\hline Total reads & $3.27 \mathrm{E}+08$ & $2.79 \mathrm{E}+08$ & $5.97 \mathrm{E}+06$ \\
\hline $\begin{array}{l}\text { Average } \\
\text { sample reads }\end{array}$ & $1.36 \mathrm{E}+07$ & $1.16 \mathrm{E}+07$ & $2.49 \mathrm{E}+05$ \\
\hline $\begin{array}{l}\text { Sample } \\
\text { minimum }\end{array}$ & $9.63 \mathrm{E}+06$ & $8.23 \mathrm{E}+06$ & $1.45 \mathrm{E}+05$ \\
\hline $\begin{array}{l}\text { Sample } \\
\text { maximum }\end{array}$ & $1.90 \mathrm{E}+07$ & $1.61 \mathrm{E}+07$ & $5.17 \mathrm{E}+05$ \\
\hline $\begin{array}{l}\text { Standard } \\
\text { deviation }\end{array}$ & $2.21 \mathrm{E}+06$ & $1.88 \mathrm{E}+06$ & $9.03 \mathrm{E}+04$ \\
\hline $\begin{array}{l}\text { Percent total } \\
\text { a Unmapped reads make up } \sim 2.1 \% \text { of total reads and were mapped to } \\
\text { the phytobiome using ParaKraken. }\end{array}$ &
\end{tabular}

quantiles of all data. Four Jaccard indices (Jaccard 1901) are created that identify mutualism/commensalism (up-up or down-down) and amensalism (up-down or down-up) relationships between taxa across sample groups. A threshold of 0.75 was used to identify the most highly interactive organisms in the phytobiome (Supplementary Table S14).

Transcriptome-phytobiome comparison. Normalized transcriptome read counts were combined with scaled phytobiome counts. Transcript and taxa counts were scaled by the maximum count for the given taxa/transcript. The scaled counts were run through DUO and postprocessed to include only significantly differentially expressed genes, significantly differentially abundant taxa, and DUO scores of $>0.75$. GO terms were mapped to transcripts and an enrichment calculated on GO terms for a given taxa using a modified Fisher's exact test (Garcia et al. 2015; Hosack et al. 2003) with Benjamini-Hochberg (Benjamini and Hochberg 1995) multiple-testing correction. The enrichment was performed separately for DUO values that were positive (up-up or down-down) and negative (down-up or up-down) with an FDR cutoff of 0.05 . MapMan annotations were also mapped to each gene in the DUO network and parsed for trends in gene function.

\section{RESULTS}

RNA sequencing depth and coverage. To identify changes to the $P$. deltoides transcriptome and phytobiome, we performed RNAseq on leaf samples of well-watered controls, acute drought days 5, 7, and 8, cyclic mild drought, and cyclic severe drought. Water potentials for each drought phase can be seen in Table 1. A total of 327 million reads were sequenced, with an average of 13.6 million reads per sample (Table 2). Reads were first aligned to the $P$. deltoides genome, with $85.5 \%$ of total reads mapping uniquely to the transcriptome, resulting in 6.83 million $(2.1 \%)$ unmapped reads. The unmapped reads were run through ParaKraken to identify other members of the phytobiome, resulting in $1.83 \%$ of all reads and $87.3 \%$ of the unmapped reads mapping to phytobiome taxa.

$\boldsymbol{P}$. deltoides transcriptome. Photosynthesis and glycolysis alterations in drought versus control. Differential expression analysis of the drought transcriptome yields 33,309 significantly differentially expressed transcripts across all drought conditions versus their respective controls (Supplementary Fig. S2). Overall, the differential expression analyses reveal similarities between acute drought and cyclic drought relative to well-watered controls, specifically in acute day 7 versus day 0 , acute day 8 versus day 0 , and cyclic severe drought versus control (Fig. 2), with the fold change of significantly differentially expressed genes being highly correlated between these samples (Fig. 3A). Moreover, the more severe drought conditions show similar significant enrichments of GO terms relative to their respective controls (Supplementary Fig. S3). For example: enrichments associated with day 7 , day 8 , and cyclic severe indicate putative downregulation of photosynthesis and photosystems, while day 5 (acute mild) indicate putative upregulation. Day 8 shows downregulation of glycolytic process, pyruvate metabolic process, fructose-bisphosphate aldolase, and fructose 1,6-bisphosphate 1-phosphatase activity; whereas, day 7 and cyclic severe only have significant downregulation of the fructose 1,6bisphosphate 1-phosphatase activity, and fructose-bisphosphate aldolase. On day 7, day 8, cyclic mild, and cyclic severe, we observed upregulation of transcription factor activity and regulation terms including nitrogen metabolic process, RNA metabolic process, gene expression, and macromolecule biosynthetic process. The increase in regulatory GO terms have been associated with decreased nitrogen metabolism (Meng et al. 2016) and altered metabolic activity during drought stress. In contrast to the other 
samples, a downregulation of the regulatory terms was observed on day 5 compared with the control.

Genes with MapMan annotations associated with stomata, chloroplast, aquaporins, and signaling molecules were commonly significantly differentially expressed in drought relative to controls. Members of AtCBLs, genes associated with potassium transport (Cheong et al. 2007; Liu et al. 2013), water deprivation (Kudla et al. 1999; Liu et al. 2013), and stomatal movement (Cheong et al. 2007), were upregulated in day 7 , day 8 , cyclic mild, and cyclic severe in response to drought; however, in day 5, AtCBL genes were significantly downregulated in response to drought. Genes annotated with chloroplast related functions had lower expression in acute drought days 7 and 8, cyclic severe drought, and cyclic mild drought (average of 2.7 times more genes down than up), while day 5 had 2.6 times more chloroplast genes with significantly higher expression than lower expression. The trend in chloroplast genes matched GO term changes in photosynthesis (except cyclic mild as the downregulation of photosynthesis was not significant after correction). Additionally, more aquaporin genes, transporters associated with water channel activity and $\mathrm{CO}_{2}$ conductance (Flexas et al. 2006), were significantly less expressed in acute drought days 7 and 8, and cyclic severe drought (average of 1.6× less genes), while aquaporin genes were significantly more expressed in cyclic mild and acute day 5 drought (average of $2.3 \times$ more genes).

Drought induced alterations to hormones and small molecules. Abscisic acid (ABA)- and cutin-related transcripts were significantly differentially expressed in drought versus control. ABA genes, which are associated both with hormones induced by drought stress and with stress tolerance (Sah et al. 2016; Tuteja 2007; Zandalinas et al. 2017), were more highly expressed in days 7 and 8 , cyclic mild, and cyclic severe (average of $2.1 \times$ more genes), while ABA-related genes were less expressed in day $5(1.9 \times$ less genes). Cutin-related genes, small lipid molecules associated with preventing water loss (Chen et al. 2011), are initially upregulated in day 5 and cyclic mild drought ( 8 genes and 6 genes upregulated, respectively), while in days 7 and 8 , and cyclic severe cutin genes are more downregulated (5 genes, 2 genes, and 3 genes downregulated, respectively, with each of the three conditions only having 1 gene upregulated).

Cyclic drought stress impacts chloroplast, reactive oxygen species (ROS) metabolism, and ABA gene expression. Despite similarities in the more severe drought conditions, there are differences between cyclic severe drought and acute drought days 7 and 8 independent of water deficit. As such, we performed differential expression analysis between these three samples to better understand the effects that cyclic drought has on the transcriptional adaptation to more severe drought conditions (Fig. 4). The differential expression analysis resulted in 18,040 genes significantly differentially expressed across the three comparisons (Supplementary Fig. S4). There are 5,244 significantly differentially expressed genes between acute days 7 and 8 resulting in the following significant GO term enrichments (Supplementary Fig. S5): day 8 versus day 7 has upregulation of phosphorus-related GO terms (phosphorylation terms and phosphorus metabolic process terms) and regulation terms: nitrogen metabolic process, RNA metabolic process, gene expression, macromolecule biosynthetic process, etc. Day 8 has a downregulation of transporter, transmembrane, and microtubules terms relative to day 7 . In a comparison of acute drought day 7 to cyclic severe and day 8 to cyclic severe drought, days 7 and 8 have downregulated ROS metabolism and phosphorus metabolic process, with day 7 having additional downregulation of photosynthesis terms relative to cyclic severe drought.

When comparing the MapMan annotations of significant genes, all three drought comparisons: day 8 versus day 7 , day 7 versus cyclic severe, and day 8 versus cyclic severe, have a slightly lower expression of chloroplast related genes $(1.2,1.3$, and 1.2 gene ratio, respectively). The direction of differentially expressed aquaporinrelated genes is also the same as the chloroplasts related genes with ratios of 3.0, 1.7, and 1.5, respectively. Days 7 and 8 have slightly higher ABA-related gene expression than cyclic severe (gene ratios of 1.3), while cyclic severe has more cutin-related genes significantly increased ( 4 genes up versus day 7 , and 3 genes up and 1 down versus day 8). The differences in both the GO terms and MapMan annotations suggest that days 7 and 8 are experiencing slightly more drought-related stress than cyclic severe drought.

Phytobiome evaluation. To further understand potential differences in the acute or cyclic nature of the drought, we profiled the unmapped transcriptome reads against a database of microorganisms, aphids, and $P$. deltoides. $P$. deltoides interacts with its phytobiome (Supplementary Fig. S6) to produce a holistic response to drought stress. As such, characterizing both the host plant and phytobiome allows for a better understanding of how the system is affected by and adapts to drought stress. Given the difference between the acute drought and cyclic drought in the $P$. deltoides transcriptome, the response in the rest of the phytobiome is also likely to be influenced by the acute or cyclic nature of the drought, due to both changes in the host and changes in the environment.

Potential beneficial taxa are more abundant in cyclic drought versus control. There were 15 significantly differentially abundant taxa in plant leaves exposed to cyclic drought relative to the wellwatered control plants. Most of the significant differentially abundant taxa between cyclic drought and control are ubiquitous

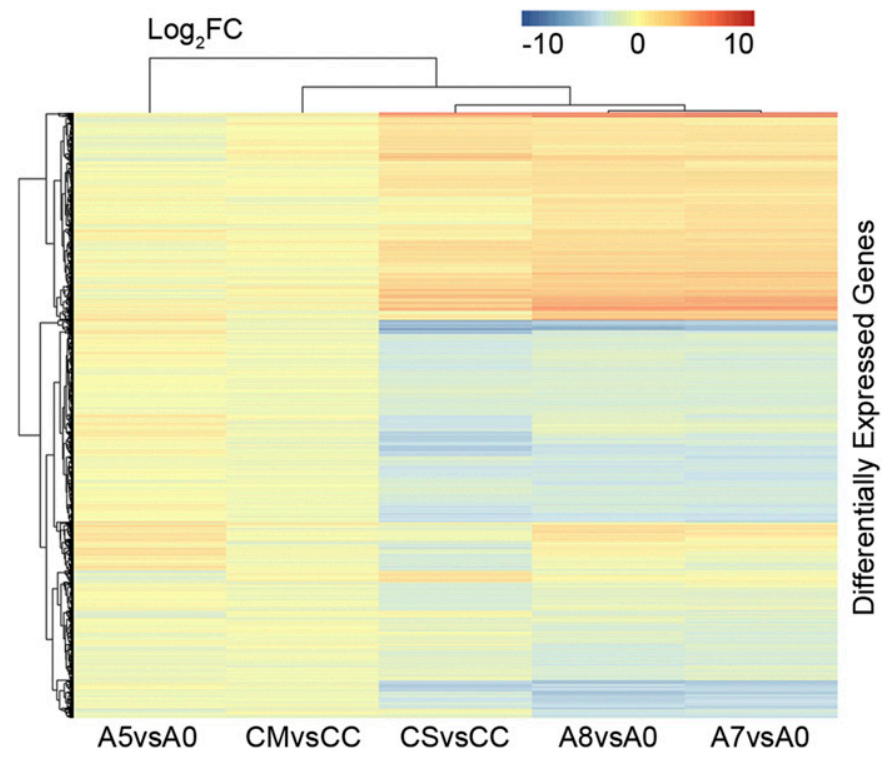

Fig. 2. Significantly differentially expressed genes across all drought versus control comparisons where each gene visualized had a $\log _{2}$-fold change greater than two in at least one comparison. Differential expression was assessed using fcros (Dembélé and Kastner 2014) and correlations were calculated using a Pearson correlation coefficient. Acute day 7 and day 8 versus day 0 were the two most correlated samples, with cyclic severe versus control having a similar profile to day 7 and day 8 versus day 0 . The more severe drought conditions had downregulation of genes involved in photosynthesis and energy metabolism. Acute day 5 versus control was dissimilar to every other sample with near opposite gene ontology term enrichments compared with more severe drought conditions. A, acute; CM, cyclic mild; CS, cyclic severe; and CC, cyclic control. 
within the environment with unknown functional implications. However, many of the taxa that are significantly more highly abundant in cyclic drought versus control are taxa mostly reported with beneficial effects to the host plant, e.g., Tricholoma (Murata et al. 2013) and Streptomyces (Qin et al. 2017; Rey and Dumas 2017). Conversely, potential pathogens and pathogen-like microorganisms have significantly higher abundance in the controls, e.g., Pseudomonas syringae and Xanthomonas vasicola.

Higher abundance of potential pathogens in acute drought versus control. There were 42 significantly differentially abundant taxa in plant leaves exposed to acute drought versus day 0 , most of them are ubiquitous in the environment with unknown functional implications. However, there was a trend of significantly increased potential pathogen-like taxa abundance in acute drought, e.g., Fusarium oxysporum (Hill et al. 2011), Alternaria alternata, and a variety of organisms with sequence similarity to a wide range of pathogens (Fig. 5). Conversely, a Pleosporales with similarity to a fungal endophyte, Periconia macrospinosa (Alberton et al. 2010; Jumpponen 2001; Mayerhofer et al. 2013) was found in significantly higher abundance in days 7 and 8. Periconia macrospinosa can promote selection mosaics, a reciprocal and spatially related increase in fitness, of fungi beneficial to plant health (Piculell et al. 2008).

Distinct differences in the phytobiome between acute drought and cyclic drought. Unlike the $P$. deltoides transcriptome, the phytobiome has a more distinct separation of significantly differentially abundant taxa between acute drought versus control and cyclic drought versus control (Figs. 3B and 5). Acute drought averaged more significant differentially abundant taxa per sample relative to control (average of 14 versus 7.5 for cyclic). There is also a slightly higher proportion of differentially abundant fungal taxa in acute drought ( $81 \%$ versus $66 \%$ in cyclic drought), and a higher

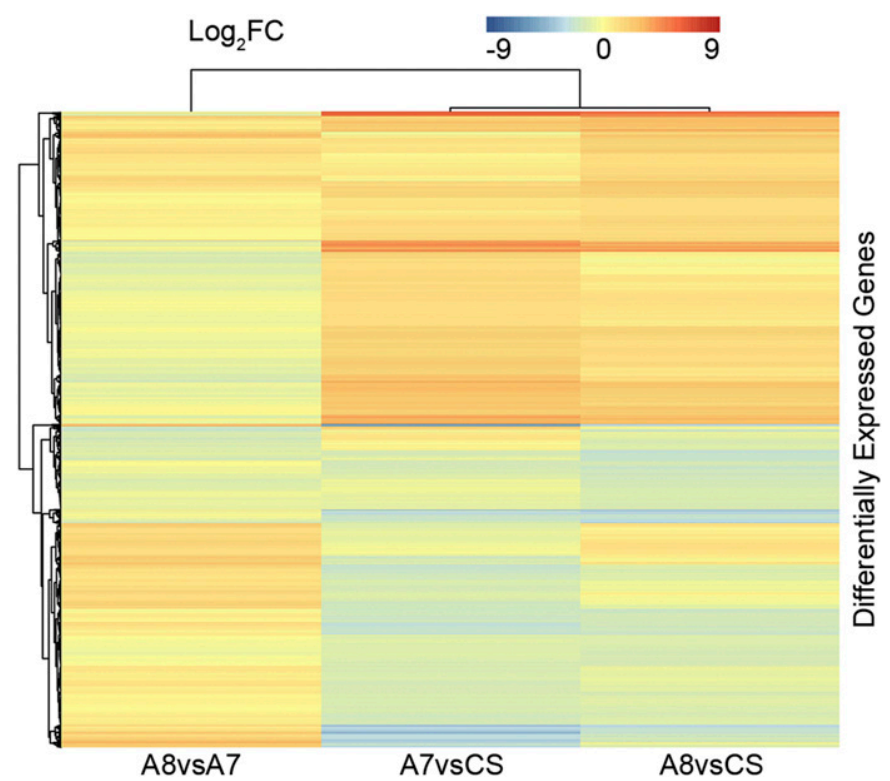

Fig. 4. Significantly differentially expressed genes from a comparison of acute day 7 , acute day 8 , and cyclic severe drought with a $\log _{2}$-fold change greater than one in any given comparison. Differential expression was assessed using fcros (Dembélé and Kastner 2014) and correlations were calculated using a Pearson correlation coefficient. Differences in expression between day 7 and day 8 were relatively smaller in comparison with cyclic severe, with significantly lower photosynthesis and reactive species metabolism in acute drought compared with severe drought. A, acute; and CS, cyclic severe.
A

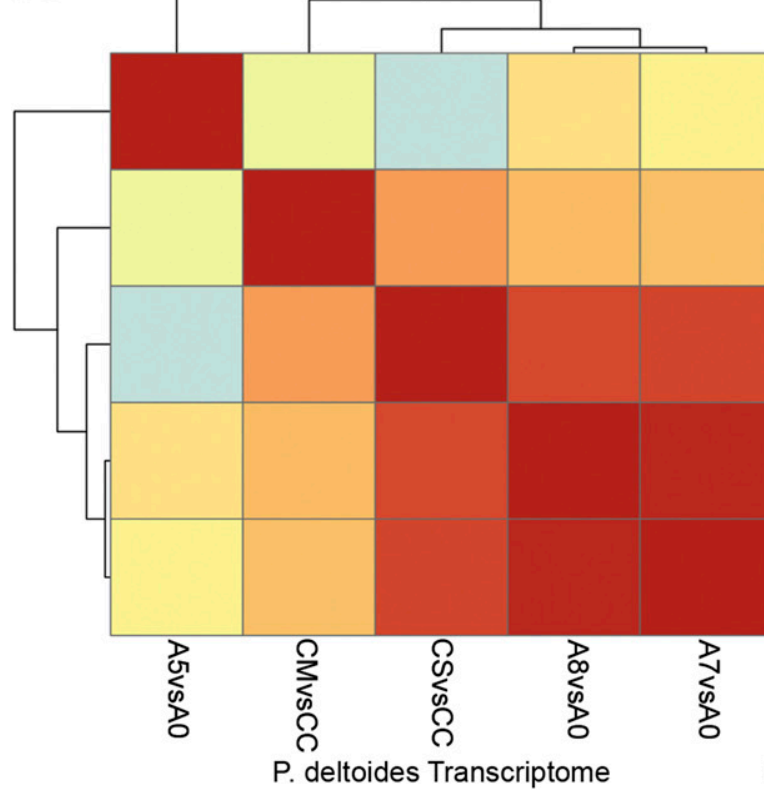

A5vsA0

CMvsCC

CSvsCC

A8vsA0

A7vsA0

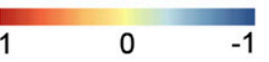

Pearson's Correlation
B

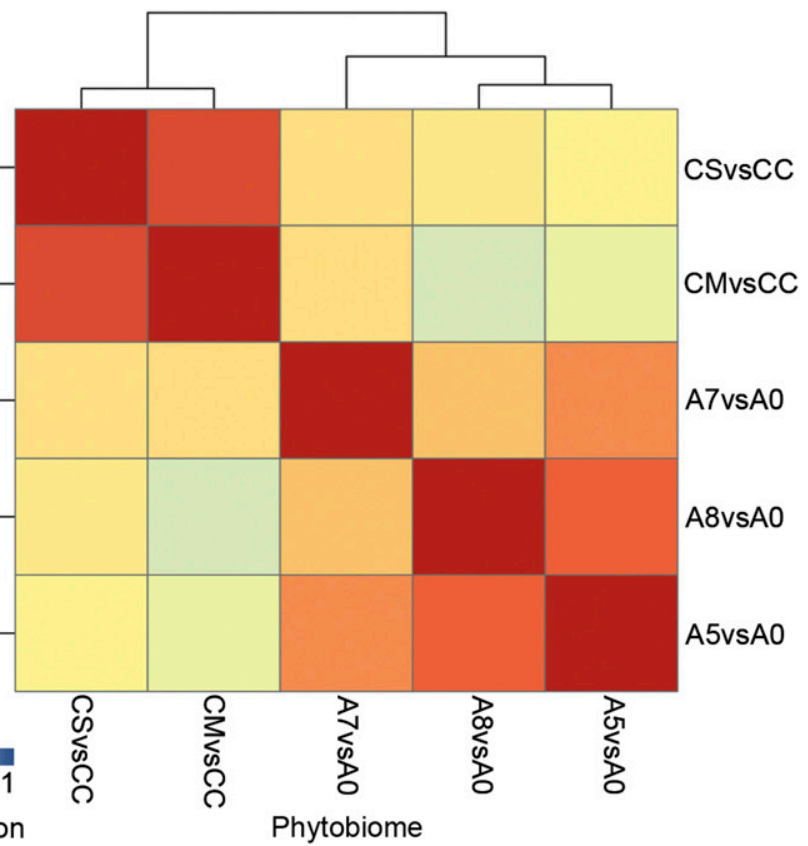

Fig. 3. Correlations and clustering of $\log _{2}$ changes of $\mathbf{A}$, significantly differently expressed genes in drought versus their respective control, and $\mathbf{B}$, significantly differently abundant species level taxa in drought versus their respective control. Hierarchical clustering was performed on Pearson correlation coefficients between samples as described in the Methods section. More severe drought conditions (day 7, day 8, cyclic severe) clustered together in the transcriptome, while there was a clear divide between acute and cyclic drought in the phytobiome suggesting that while water deficit is driving a lot of the changes in the transcriptome, the cyclic nature of the drought had a larger effect on the phytobiome. It is likely that day 5 and day 8 are more similar to each other in the phytobiome relative to day 7 as day 5 and day 8 samples came from the same plant. A, acute; CM, cyclic mild; CS, cyclic severe; and CC, cyclic control. 
proportion of all taxa upregulated in acute versus cyclic drought (76\% versus $67 \%$, respectively).

Similar to the plant transcriptome comparison, we also compared day 7 , day 8 , and cyclic severe to identify potential changes in the phytobiome that are independent of water deficit. A comparison of acute day 7 to acute day 8 , acute day 8 to cyclic severe, and acute day 7 to cyclic severe drought resulted in 18 (day 8 versus 7), 44 (day 8 versus severe), and 43 (day 7 versus severe) significant differentially abundant taxa (Fig. 6). The proportion of significantly differential taxa that are fungal taxa was $78 \%$ (day 8 versus 7 ), $70 \%$ (day 8 versus severe), and $67 \%$ (day 7 versus severe). Of the 44 significantly differentially abundant species in day 8 versus severe, 30 of these taxa were also significantly differentially abundant in day 7 versus severe.

We observed a significantly higher abundance of reported taxa with beneficial traits in root systems in cyclic severe drought versus acute drought days 7 and 8, e.g., Rhizophagus and Streptomyces sp. 3. It is unknown whether or not the prevalence of root organisms and ectomycorrhizal fungi in our leaf samples is due to database bias or due to colonization of the leaves by organisms originally isolated in roots. Streptomyces is mostly reported for its ability to suppress fungal and bacterial infections (de Lima Procópio et al. 2012). Additionally, Mesorhizobium was significantly more abundant in cyclic severe drought, a taxa mostly reported for nitrogen fixation in soil (Vurukonda et al. 2016). Similarly, Rhizophagus is a mutualistic soil fungal taxa commonly associated with plant nutrition and drought tolerance (Zhang et al. 2017; Ruiz-Lozano et al. 2016). Rhizophagus has been associated with decreasing the damage of oxidative stress through alternative oxidase activity (Fuentes et al. 2016; He et al. 2017; Miller et al. 2008), increasing aquaporin expression (He et al. 2016), and increasing potassium availability (Deepika and Kothamasi 2014). Additionally, Rhizophagus and Streptomyces are reported to have beneficial interactions in plants (Abdel-Fattah and Mohamedin 2000), including Rhizophagus mitigating drought stress in Populus canadensis (Liu et al. 2015). Conversely, there were higher abundances of potential pathogens in acute drought than in cyclic severe conditions, e.g., Fusarium (Hill et al. 2011), Magnaporthe (Sesma and Osbourn 2004), Pseudomonas (Munsch et al. 2000), and Zymoseptoria (Gyenis et al. 2003; McDonald et al. 2015).

Relationships between species in the phytobiome. Potential symbiotic relationships (interactions) between organisms in the phytobiome were established using DUO, an algorithm that identifies potential mutualism/commensalism and amensalism by calculating Jaccard coefficients between high and low abundances between two taxa. Of the most highly interacting organisms (score > 0.75), two are nematodes, 12 bacteria, and 13 fungi (Fig. 7). The most interactive fungal species are Rutstroemia and Rhizophagus. Both of the taxa are higher in cyclic severe drought versus acute drought. Rhizophagus has a mixture of mutualistic/commensal and amensalistic relationships within the network. All of the amensalistic relationships between Rhizophagus and other taxa are shared with the nematode (Trichinella sp. 1; Rhizophagus has a mutualistic/commensal relationship with the nematode based upon DUO scores). Of the interacting species, the nematode has the highest degree of interactivity, with $64 \%$ being mutualistic/ commensal/parasitic. Though it is unknown whether this nematode is a plant parasite or benign to the plant, many nematodes are able to suppress host immune responses (Lin et al. 2016).

The bacterium with the most interactions was the Mesorhizobium species, the same organism with significantly increased abundance in cyclic severe versus acute days 7 and 8. Within the Mesorhizobium interactive network, 8 of 11 interactions are predicted to be mutualistic/commensal (with all five fungal interactions in the positive association group). Mesorhizobium, Rhizophagus, and Trichinella sp. 1 form a triangle of mutualistic/commensal interactions that is highly connected to other taxa within the network. The bacterium with the second most abundant number of interactions is Streptomyces sp. 3, which has a predicted mutualistic/commensal interaction with the most interactive nematode (Trichinella sp. 1) and amensalistic interactions with Trichinella sp. 2. Streptomyces has mixed associations with nematodes, with some Streptomyces species increasing the growth of nematodes that consume fungus/plant tissues and other Streptomyces species decreasing the growth of nematodes that consume bacteria (Ma et al. 2017). Streptomyces sp. 3 also has four predicted mutualistic/commensal interactions with fungi, one amensalistic fungus relationship, one mutualistic/commensal bacteria relationship, and one amensalistic bacteria relationship in the phytobiome.

Relationships between differential transcripts and differential taxa. Correlations between transcriptional abundance and species abundance were established using DUO with a score of $>0.75$. GO term enrichments were performed on positive gene associations with taxa and negative gene associations with taxa separately (Supplementary Table S15 and S16 and Fig. 8). Genes with taxa associations were also annotated with the MapMan annotations. ROS metabolic and superoxide metabolic GO terms were positively associated with abundances of six significantly different taxa, including Rhizophagus, Streptomyces, and Trichinella. As previously mentioned, Rhizophagus has been associated with decreased oxidative stress (Fuentes et al. 2016; He et al. 2017; Miller et al. 2008). Additionally, ROS are beneficial for mitigating nematode infection (Beneventi et al. 2013; Nath et al. 2017).

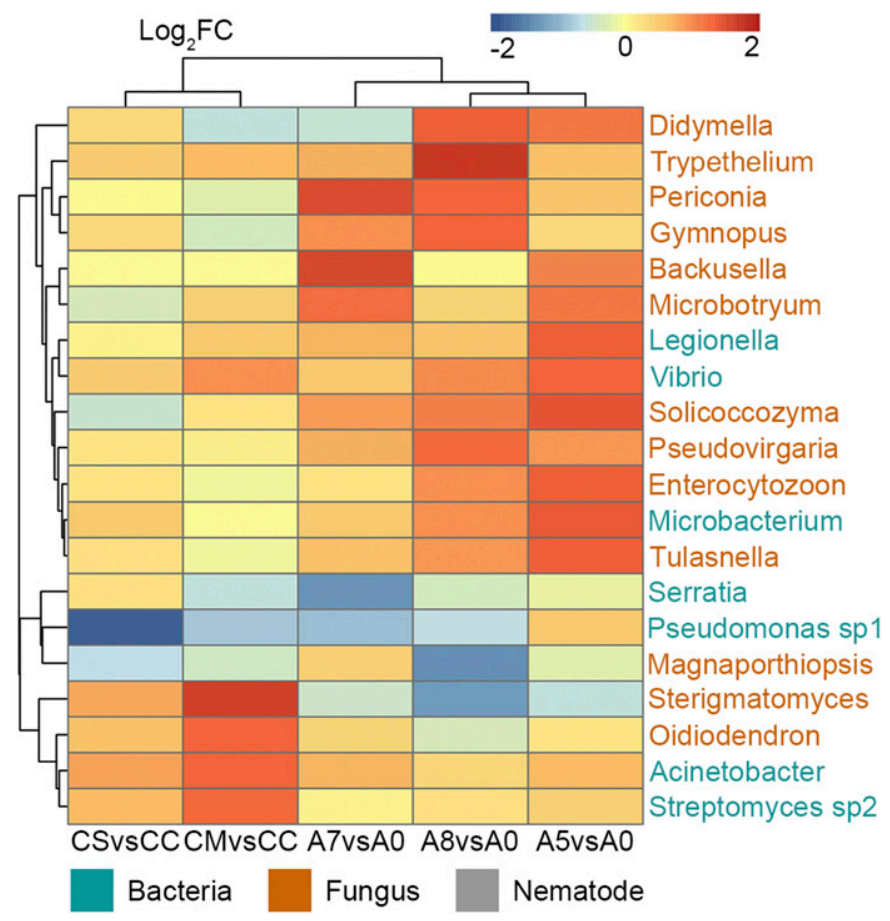

Fig. 5. Top 20 significant species, named by most closely associated genus, comparing drought to control with a $\log _{2}$-fold change greater than one in at least one comparison. Differential abundance was assessed using fcros (Dembélé and Kastner 2014) and correlations were calculated using a Pearson correlation coefficient There was a separation in the phytobiome between acute and cyclic drought, with the majority of significant species being composed of fungi and a wide variety of ubiquitous organisms with unknown effects on plants. A, acute; $\mathrm{CM}$, cyclic mild; CS, cyclic severe; and CC, cyclic control. 
Genes with MapMan annotations, such as disease resistance, response to dehydration and water deprivation, and photosynthesis and photosystem, were associated with taxa in the phytobiome. Early response to dehydration is positively associated with five taxa that are significantly more abundant in acute drought (Malassezia, Klebsiella, Phycomyces, Pseudomonas, and Thielavia) and four taxa that are significantly more abundant in cyclic severe drought (Rutstroemia, Brettanomyces, Conidiobolus, and Puccinia). However, genes associated with general water deprivation were positively associated with seven taxa (the above four plus Trichinella, Streptomyces, and Mesorhizobium) that were only significantly more abundant in cyclic drought. Additionally, 22 genes annotated with disease resistance were associated with ten taxa that were significantly more abundant in cyclic drought (Rutstroemia, Trichinella, Puccinia, Streptomyces, Brettanomyces, Atribacteria, Rhizophagus, Aspergillus, Mesorhizobium, and Condidiobolus) and 21 genes were associated with five taxa that were significantly more abundant in acute drought (Malassezia, Klebsiella, Phycomyces, Pseudomonas, and Thielavia). There is a lower percent of positive interactions in cyclic taxa compared with acute taxa ( $81 \%$ versus $96 \%$, respectively) with only two unique genes $(5 \%)$ shared between acute taxa and cyclic taxa.

Photosynthesis-related transcripts were significantly affected by drought and the cyclic nature of the drought, as seen in the differential abundance, and are also positively associated with five taxa that are significantly more abundant in acute drought (Malassezia, Klebsiella, Phycomyces, Pseudomonas, and Thielavia) and six taxa that are significantly more abundant in cyclic drought (Rutstroemia,

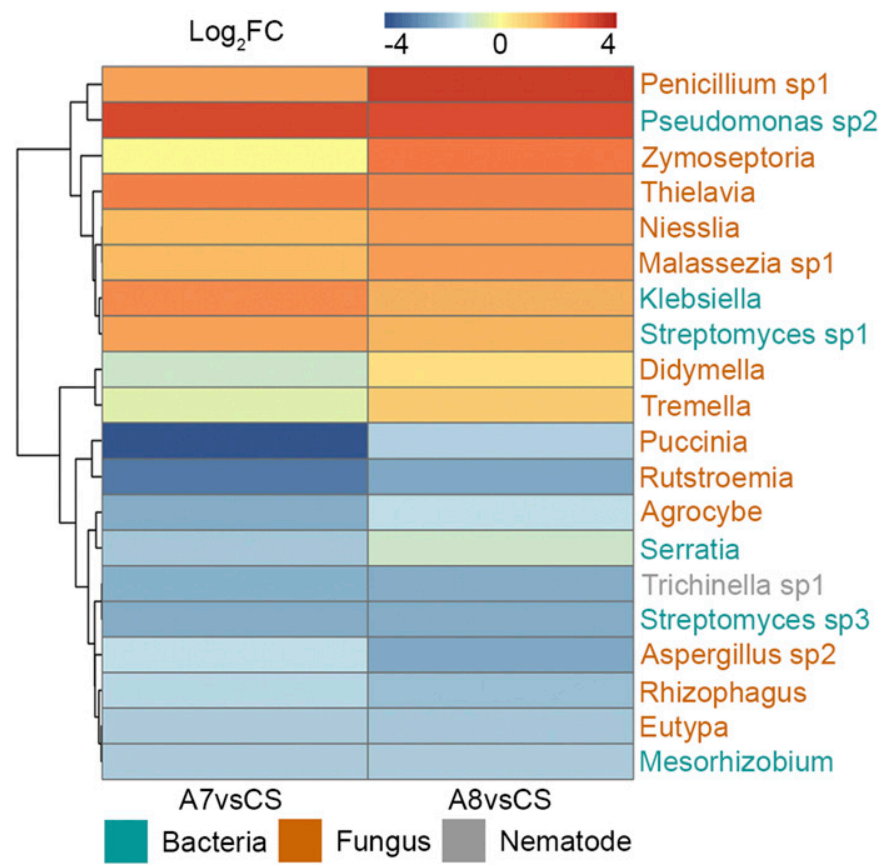

Fig. 6. Top 20 significant species, named by most closely associated genus, comparing acute day 7 , acute day 8 , and cyclic severe drought with a $\log _{2}$-fold change greater than one in at least one comparison. Differential abundance was assessed using fcros. Genera with multiple significant species are labeled with their species ID. Similar to drought versus control comparisons, differentially abundant taxa in the more severe drought were mostly fungi. Genera with reported plant pathogens were higher in abundance in day $7 / 8$, while genera with reported potential drought beneficial organisms and plant growth promoters were higher in cyclic drought, though it is unknown if the taxa differentially abundant in severe drought had any pathogenic/beneficial effects. A, acute; and CS, cyclic severe.
Trichinella, Puccinia, Streptomyces, Brettanomyces, and Conidiobolus). Similar to associations with disease resistance and dehydration discussed above, there are differences in photosynthesis associations between acute drought and cyclic drought taxa. Acute drought taxa are positively associated with both photosystem I and photosystem II genes, while cyclic drought taxa are positively associated with photosystem II and phosphofructokinase photosynthesis-related genes. Pseudomonas, a taxa significantly more abundant in acute drought that has positive associations with both photosystem I and II, has been previously shown to have associations with photosystem II activity in Populus (Khan et al. 2016); though, photosynthesis associations appear to be strain and condition specific within the genus Populus (Knoth et al. 2014).

\section{DISCUSSION}

Marginal land represents potential areas to grow economically important plants, such as $P$. deltoides, without displacing food crops in high fertility and low drought stress areas; however, these areas represent additional challenges to plant growth that need to be overcome to make them economically viable (Jones et al. 2015). Such marginal lands experience high variability in rainfall and concomitant drought stress (Shahid and Al-Shankiti 2013), so drought experiments need to reflect the cyclic nature of droughts experienced in marginal lands. In this experiment, we subjected plants to both acute and cyclic droughts to identify the effects of

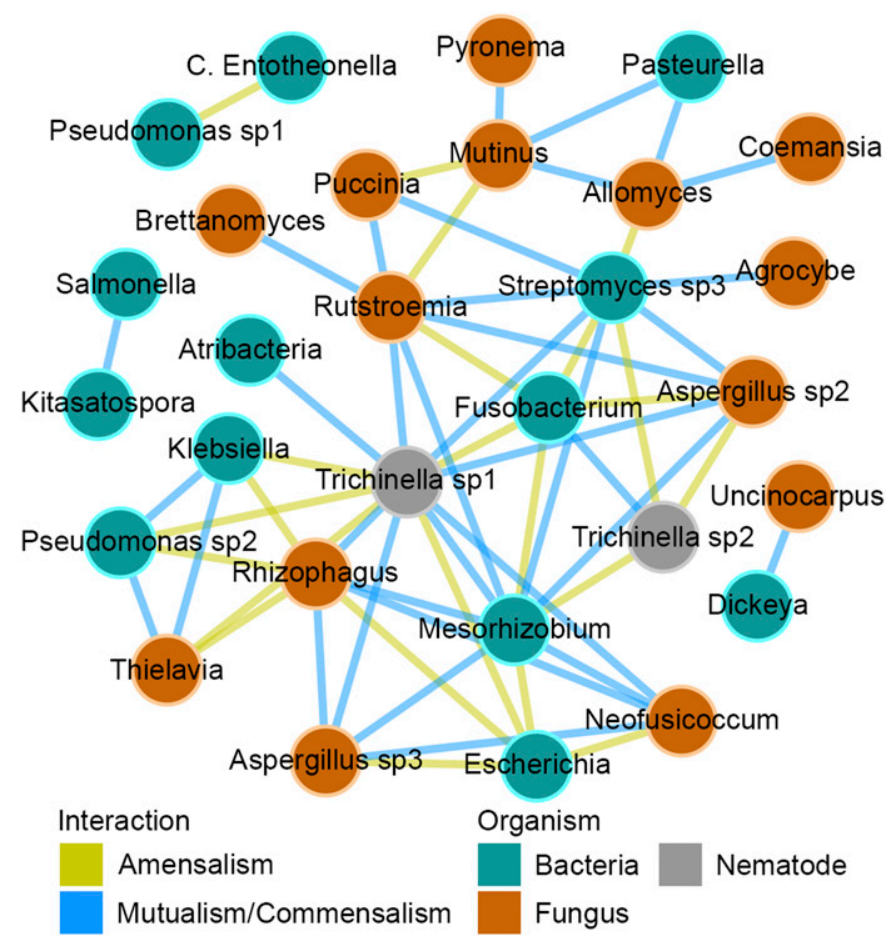

Fig. 7. Potential amensalism and mutualism/commensalism associations between members of the phytobiome. Associations were established using DUO with species abundances (named by most closely associated genus and species number for genus with multiple species) across all conditions. Similar to other phytobiome analyses, fungi also make up the majority of species with significant interactions. The nematode, Trichinella sp. 1, was the most interactive species, potentially due to the organism either suppressing the host immune system or consuming other members of the phytobiome. Rhizophagus and Mesorhizobium were also highly interactive, one genus was associated with increasing potassium and phosphorus availability while the other was associated with nitrogen fixation. 
drought at different water deficits and to identify any changes that occur by subjecting the plants to multiple cycles of drought. Drought in Populus has a wide range of effects that include altering (i) ROS metabolism and chlorophyll protein translation (Abraham et al. 2018), (ii) stomatal patterning and leaf size (Viger et al. 2016), (iii) photosynthesis-related and carbohydrate metabolism-related transcription (Cossu et al. 2014; Hamanishi et al. 2015; Tang et al. 2015), and (iv) microbiomes that may both affect plant physiology and may be affected by changes in plant physiology (Timm et al. 2018). However, to our knowledge, no plant-based drought studies have leveraged RNAseq for both identifying changes in plant and phytobiome in the same samples.

While our study was conducted in a greenhouse setting, our Populus transcriptome results, including photosynthesis, carbohydrate, and ROS transcriptional changes, match drought studies conducted in other settings from both Populus and other plant genera. Similarities with prior studies allow us to establish general host changes and hypotheses for Populus drought that may be extendable beyond the greenhouse. However, phytobiome changes are less well known in drought, especially in leaf systems and in differences between greenhouse and field. Additionally, phytobiome characterization is only as good as the databases utilized (16S and other marker sequencing also suffers from the limitation), meaning accuracy increases with an increasing number of genomes (especially genomes isolated from similar systems). Taxa classification represents closest ancestor or relative in many cases. Despite the limitation, we identified similarities with other phytobiome systems both in Populus and other genera, similarities to root systems, and relationships between host transcription and taxa that are supported by literature.

In the $P$. deltoides transcriptome, water deficit is a main driving factor of the plant response to drought stress, with prior drought exposure having an impact as well. There is a marked difference in response to drought between day 5 (acute mild), cyclic mild, and more severe drought (acute day 7, acute day 8, cyclic severe), related to the multiple stages of drought with severe drought resulting in metabolic impairment (Flexas et al. 2006). Day 5 has the lowest water deficit and is the only sample with significantly increased photosynthesis-related terms. Cyclic mild falls in between day 5 and day 7 in water deficit and shares a subset of downregulated metabolic changes and a subset of stomatal genes with more severe droughts. Cyclic mild also shares reduced expression of chloroplast-related genes with more severe drought conditions; however, cyclic mild and acute day 5 share an increase in aquaporin- and cutin-related genes, suggesting that the drought experienced in the cyclic mild treatment does not cause as much metabolic inhibition as more severe drought conditions. Acute day 7 , acute day 8 , and cyclic severe drought are highly correlated and share similar downregulation of transcripts associated with photosynthesis, chloroplasts, and aquaporins. Changes that occur in more severe drought conditions (acute day 7, acute day 8, and cyclic severe) suggest that water deficit is a large driver of transcriptional changes in severe drought that ultimately results in metabolic impairment. Populus protein translation mirrors the transcriptome showing changes in stomata, chlorophyll, and carbohydrate metabolism that are dependent both on the intensity and cyclic nature of drought (Abraham et al. 2018). Photosynthesis and energy metabolism transcription are largely decreased in other Populus species during drought (Cossu et al. 2014; Tang et al. 2015), though drought-resistant species are able to mitigate much of the photosynthesis suppression (Tang et al. 2015). Additionally, decreased photosynthesis (Zandalinas et al. 2017) and altered glycolysis (Pan et al. 2016) are general responses for plants to drought stress.

Despite cyclic severe drought water potential falling in between days 7 and 8 of acute drought, there are potential adaptations caused by cyclic drought exposure that may allow cyclic drought plants to be better suited to drought, even under metabolic impairment. Cyclic severe drought has significantly higher photosynthesis and ROS metabolism transcripts relative to acute day 7 and day 8 , which is also mirrored in the proteome (Abraham et al. 2018). Increased ROS metabolism is associated with stress tolerance and increased photosynthesis during stress (Zandalinas et al. 2017), and suggests an adaptive response during cyclic drought. The higher abundance of Rhizophagus, which is associated with alternate oxidase activity and has a positive DUO association with host ROS metabolic genes, may also decrease oxidative stress in plant leaves (Supplementary Fig. S7) (Campos et al. 2015). Cyclic severe drought also has higher expression of aquaporins, which is potentially influenced by the higher abundance of Rhizophagus (He et al. 2016). Interestingly, Rhizophagus has been associated with higher water-use efficiency in Populus root systems (Liu et al. 2015), further supporting the premise that Rhizophagus may be beneficial under drought conditions in leaf systems. The differences between severe cyclic drought and acute drought in both the transcriptome and phytobiome indicate that $P$. deltoides individuals that have been exposed to cyclic severe drought may be better acclimated to drought conditions.

Unlike the transcriptomic changes that largely track water potentials, the changes in the phytobiome displayed more distinct differences between acute and cyclic drought. For example, cyclic drought has higher abundances of some microorganisms, such as Rhizophagus, Mesorhizobium, and Streptomyces, which are usually reported to promote drought tolerance through increasing potassium

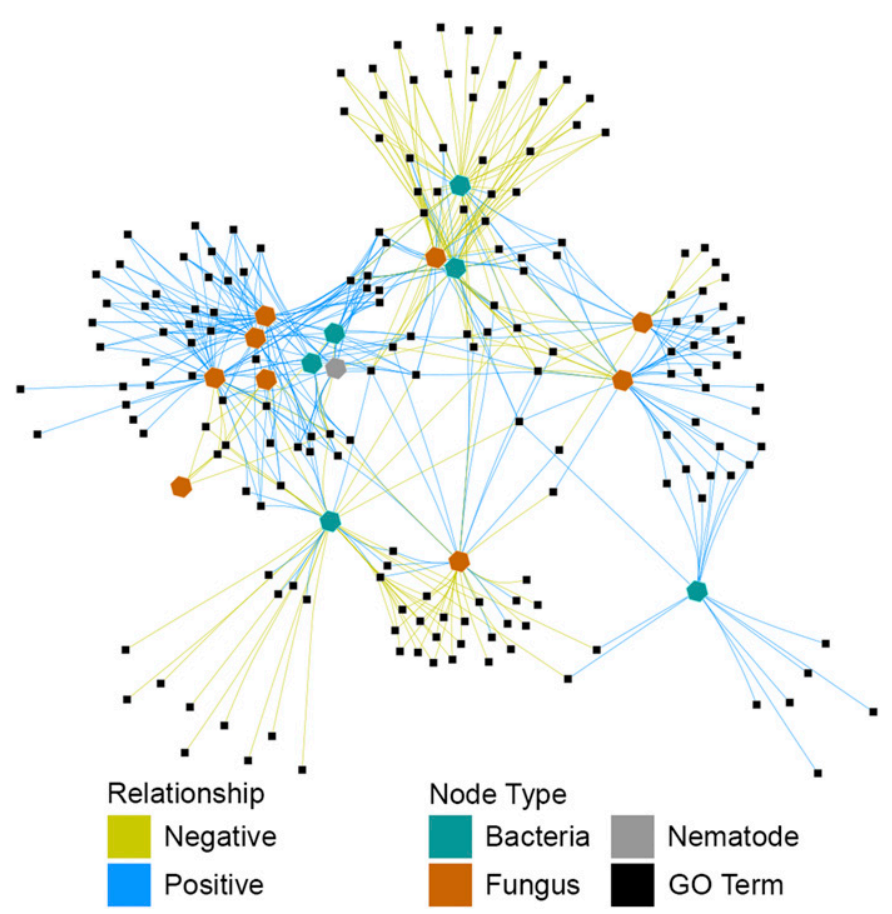

Fig. 8. Interaction network of gene ontology (GO) terms and their associated phytobiome taxa. Relationships between significantly differentially expressed genes and significantly differentially abundant taxa in any of the drought comparisons were established using DUO. GO terms associated with each gene were then used to calculate modified Fisher's exact test enrichments in order to identify patterns of functional changes positively associated with taxa and negatively associated with taxa. Rhizophagus and Trichinella were positively associated with reactive species metabolism, giving support to the hypothesis that Rhizophagus may decrease oxidative stress in the plant host while the nematode may have effects on the host immune system. 
(Zhang et al. 2017) and phosphorus availability (Deepika and Kothamasi 2014), altering plant morphology and stress response (Vurukonda et al. 2016), promoting plant growth (Sousa and Olivares 2016; Yandigeri et al. 2012), and protection from pathogens (Sousa and Olivares 2016). Streptomyces and Trichinella are also positively associated with disease resistance genes, while Streptomyces, Trichinella, and Rhizophagus are positively associated with ROS metabolism genes, suggesting that both alteration of stress response and disease response may be occurring in this study. In addition to being significantly higher abundance in cyclic mild drought versus control and cyclic severe drought versus acute drought, Streptomyces and its phylum, Actinobacteria, have been shown to have higher OTU abundance under drought stress in root systems for Populus (Timm et al. 2018), rice (Santos-Medellín et al. 2017), and grass (Naylor et al. 2017), suggesting that Streptomyces/ Actinobacteria are altered as a general response to drought stress. Conversely to cyclic drought, there is a higher abundance of pathogen-like species in acute drought, suggestive of a potential selective process happening in cyclic drought that may benefit the plant, while responses in acute drought may be indicative of opportunistic organisms exploiting the differences in carbon availability with potential fatal implications on the host's foliage.

Across experimental conditions, we have identified DUO associations between taxa suggesting interactions within the phytobiome. Streptomyces, a taxa associated with production of a wide array of antimicrobials (de Lima Procópio et al. 2012), is in the top five most interactive taxa. It is possible that the Streptomyces is providing niches for fungi and bacteria unaffected by anticipated antifungal/antibacterial compounds. Two of the top five most interactive taxa, Rhizophagus and Mesorhizobium, have been associated with increased potassium (Deepika and Kothamasi 2014) and phosphorus availability (Zhang et al. 2017) and nitrogen availability (Vurukonda et al. 2016). The high interactivity of Mesorhizobium and Rhizophagus is suggestive that they may also be providing vital nutrients to many organisms in the phytobiome. Both taxa have interactions with the most highly interactive taxa: Trichinella sp. 1, potentially through either increasing nutrient availability necessary for the nematode's growth or the growth of nematode's food source. Nematodes are able to alter and suppress the host immune response (Lin et al. 2016). Additionally, different species of nematodes are able to consume a wide array of different organisms within the phytobiome, including plant tissue (Ma et al. 2017). The highly interactive nature of Trichinella, Mesorhizobium, Streptomyces, and Rhizophagus results in an interactive web of organisms that may have an impact on the abundance and diversity of organisms growing on the plant leaves (Supplementary Fig. S8).

It is clear that plant biotic interactions, coupled with abiotic stress, affect the outcome of stress on both the plant and its phytobiome. However, there are competing mutualism, commensalism, and parasitism interactions where host-microbiome associations result in promoting fungal interactions necessary for survival, while also potentially encouraging parasitism that results in the death of the host plant. The continuum of mutualism to parasitism is influenced by the diversity and composition of the phytobiome (Grünig et al. 2008; Mandyam et al. 2012, 2013; Munkvold et al. 2004), the diversity and functional characteristics of the host (Hoeksema et al. 2010; Karst et al. 2009), and the abiotic variability of stress (Johnson 2010; Rodriguez et al. 2008). Coupled together, the biotic and abiotic factors result in competition within the phytobiome that drives both positive and negative responses to stress, in addition to influencing the response of the plant to stress and the effect of the stress on the plant.

In summary, both the phytobiome and host can be categorized and compared using a single RNAseq run on a given sample, allowing for novel identification of associations between taxa and the host plants. We have accomplished the dual identification of host and phytobiome by creating a pipeline that couples traditional host transcriptomics with a parallelized method to identify kmers from tens of thousands of species across all kingdoms of life (ParaKraken). In this study, we have identified that effects of drought on both the plant and phytobiome are dependent on the severity and prior drought exposure, with more severe drought causing severe metabolic impairment. However, despite potential metabolic impairment, cyclic severe drought plants have relatively higher transcription of genes involved in photosynthesis and ROS metabolism than severe acute drought. The changes suggest that cyclic drought may promote some acclimatization to drought stress even under severe drought conditions. Additionally, phytobiome changes in taxa that have been associated with nitrogen production, potassium and phosphorus uptake, and lower plant oxidative stress also suggest that cyclic drought may promote growth of taxa with putative reported benefits to the host plant. Conversely, plants that have undergone severe acute drought stress have an increase in taxa in the phytobiome that may be associated with opportunistic growth or potential pathogens. Further studies need to be performed to elucidate whether changes, and associations between the changes, that occur in both the transcriptome and phytobiome during cyclic drought allow for better acclimation to drought stress than changes that occur during progressive acute drought. Additionally, further studies need to be performed to ascertain to what extent stereotypical root organisms can colonize leaves and to further expand the number of whole genome sequences to include more leaf isolates to improve accuracy of phytobiome studies in less studied systems.

\section{LITERATURE CITED}

Abdel-Fattah, G. M., and Mohamedin, A. H. 2000. Interactions between a vesicular-arbuscular mycorrhizal fungus (Glomus intraradices) and Streptomyces coelicolor and their effects on sorghum plants grown in soil amended with chitin of brawn scales. Biol. Fertil. Soils 32:401-409.

Abraham, P. E., Garcia, B. J., Gunter, L. E., Jawdy, S. S., Engle, N., Yang, X., et al. 2018. Quantitative proteome profile of water deficit stress responses in eastern cottonwood (Populus deltoides) leaves. PLoS One 13:e0190019.

Alberton, O., Kuyper, T. W., and Summerbell, R. C. 2010. Dark septate root endophytic fungi increase growth of Scots pine seedlings under elevated $\mathrm{CO}_{2}$ through enhanced nitrogen use efficiency. Plant Soil 328:459-470.

Allen, C. D., Macalady, A. K., Chenchouni, H., Bachelet, D., McDowell, N., Vennetier, M., et al. 2010. A global overview of drought and heat-induced tree mortality reveals emerging climate change risks for forests. For. Ecol. Manage. 259:660-684.

Anderegg, W. R. L., Berry, J. A., Smith, D. D., Sperry, J. S., Anderegg, L. D. L., and Field, C. B. 2012. The roles of hydraulic and carbon stress in a widespread climate-induced forest die-off. Proc. Natl. Acad. Sci. 109:233-237.

Barghini, E., Cossu, R. M., Cavallini, A., and Giordani, T. 2015. Transcriptome analysis of response to drought in poplar interspecific hybrids. Genom. Data 3:143-145.

Beneventi, M. A., da Silva, O. B., de Sá, M. E. L., Firmino, A. A. P., de Amorim, R. M. S., Albuquerque, É. V. S., et al. 2013. Transcription profile of soybean-root-knot nematode interaction reveals a key role of phytohormones in the resistance reaction. BMC Genomics 14:322.

Benjamini, Y., and Hochberg, Y. 1995. Controlling the false discovery rate: A practical and powerful approach to multiple testing. J. R. Stat. Soc. B 57:289-300.

Bidzinski, P., Ballini, E., Ducasse, A., Michel, C., Zuluaga, P., Genga, A., et al. 2016. Transcriptional basis of drought-induced susceptibility to the rice blast fungus Magnaporthe oryzae. Front. Plant Sci. 7:e1005457.

Campos, C., Cardoso, H., Nogales, A., Svensson, J., Lopez-Ráez, J. A., Pozo, M. J., et al. 2015. Intra and inter-spore variability in Rhizophagus irregularis AOX gene. PLoS One 10:e0142339.

Chen, G., Komatsuda, T., Ma, J. F., Li, C., Yamaji, N., and Nevo, E. 2011. A functional cutin matrix is required for plant protection against water loss. Plant Signal. Behav. 6:1297-1299. 
Cheong, Y. H., Pandey, G. K., Grant, J. J., Batistic, O., Li, L., Kim, B. G., et al. 2007. Two calcineurin B-like calcium sensors, interacting with protein kinase CIPK23, regulate leaf transpiration and root potassium uptake in Arabidopsis. Plant J. 52:223-239.

Cossu, R. M., Giordani, T., Cavallini, A., and Natali, L. 2014. High-throughput analysis of transcriptome variation during water deficit in a poplar hybrid: A general overview. Tree Genet. Genomes 10:53-66.

de Lima Procópio, R. E., da Silva, I. R., Martins, M. K., de Azevedo, J. L., and de Araújo, J. M. 2012. Antibiotics produced by Streptomyces. Braz. J. Infect. Dis. 16:466-471.

Deepika, S., and Kothamasi, D. 2014. Soil moisture-A regulator of arbuscular mycorrhizal fungal community assembly and symbiotic phosphorus uptake. Mycorrhiza 25:67-75.

Dembélé, D., and Kastner, P. 2014. Fold change rank ordering statistics: A new method for detecting differentially expressed genes. BMC Bioinformatics $15: 14$.

Dobin, A., Davis, C. A., Schlesinger, F., Drenkow, J., Zaleski, C., Jha, S., et al. 2013. STAR: Ultrafast universal RNA-seq aligner. Bioinformatics 29:15-21.

Farooq, M., Wahid, A., Kobayashi, N., Fujita, D., and Basra, S. M. A. 2009. Plant drought stress: Effects, mechanisms and management. Agron. Sustain. Dev. 29:185-212.

Flexas, J., Bota, J., Galmés, J., Medrano, H., and Ribas-Carbó, M. 2006. Keeping a positive carbon balance under adverse conditions: Responses of photosynthesis and respiration to water stress. Physiol. Plant. 127:343-352.

Fuentes, A., Almonacid, L., Ocampo, J. A., and Arriagada, C. 2016. Synergistic interactions between a saprophytic fungal consortium and Rhizophagus irregularis alleviate oxidative stress in plants grown in heavy metal contaminated soil. Plant Soil 407:355-366.

Garcia, B. J., Datta, G., Davidson, R. M., and Strong, M. 2015. MycoBASE: Expanding the functional annotation coverage of mycobacterial genomes. BMC Genomics 16:1102.

Grünig, C. R., Queloz, V., Sieber, T. N., and Holdenrieder, O. 2008. Dark septate endophytes (DSE) of the Phialocephala fortinii s.1-Acephala applanata species complex in tree roots: Classification, population biology, and ecology. Botany 86:1355-1369.

Gyenis, L., Anderson, N. A., and Ostry, M. E. 2003. Biological control of Septoria leaf spot disease of hybrid poplar in the field. Plant Dis. 87:809-813.

Hamanishi, E. T., Barchet, G. L. H., Dauwe, R., Mansfield, S. D., and Campbell, M. M. 2015. Poplar trees reconfigure the transcriptome and metabolome in response to drought in a genotype- and time-of-day-dependent manner. BMC Genomics 16:329.

He, F., Sheng, M., and Tang, M. 2017. Effects of Rhizophagus irregularis on photosynthesis and antioxidative enzymatic system in Robinia pseudoacacia L. under drought stress. Front. Plant Sci. 8:e63930.

He, F., Zhang, H., and Tang, M. 2016. Aquaporin gene expression and physiological responses of Robinia pseudoacacia L. to the mycorrhizal fungus Rhizophagus irregularis and drought stress. Mycorrhiza 26:311-323.

Hill, A. L., Reeves, P. A., Larson, R. L., Fenwick, A. L., Hanson, L. E., and Panella, L. 2011. Genetic variability among isolates of Fusarium oxysporum from sugar beet. Plant Pathol. 60:496-505.

Hoeksema, J. D., Chaudhary, V. B., Gehring, C. A., Johnson, N. C., Karst, J., Koide, R. T., et al. 2010. A meta-analysis of context-dependency in plant response to inoculation with mycorrhizal fungi. Ecol. Lett. 13:394-407.

Hosack, D. A., Dennis, G., Jr., Sherman, B. T., Lane, H. C., and Lempicki, R. A. 2003. Identifying biological themes within lists of genes with EASE. Genome Biol. 4:R70.

Jaccard, P. 1901. Étude comparative de la distribution florale dans une portion des Alpes et des Jura. Bull. del la Société Vaudoise des Sci. Nat. 37:547-579.

Jiang, H., Lei, R., Ding, S.-W., and Zhu, S. 2014. Skewer: A fast and accurate adapter trimmer for next-generation sequencing paired-end reads. BMC Bioinformatics 15:182.

Johnson, N. C. 2010. Resource stoichiometry elucidates the structure and function of arbuscular mycorrhizas across scales. New Phytol. 185:631-647.

Jones, M. B., Finnan, J., and Hodkinson, T. R. 2015. Morphological and physiological traits for higher biomass production in perennial rhizomatous grasses grown on marginal land. GCB Bioenergy. 7:375-385.

Jumpponen, A. 2001. Dark septate endophytes-Are they mycorrhizal? Mycorrhiza 11:207-211.

Karst, J., Jones, M. D., and Turkington, R. 2009. Ectomycorrhizal colonization and intraspecific variation in growth responses of lodgepole pine. Plant Ecol. 200:161-165.

Khan, Z., Rho, H., Firrincieli, A., Hung, S. H., Luna, V., Masciarelli, O., et al. 2016. Growth enhancement and drought tolerance of hybrid poplar upon inoculation with endophyte consortia. Curr. Plant Biol. 6:38-47.
Kim, D., Song, L., Breitwieser, F. P., and Salzberg, S. L. 2016. Centrifuge: Rapid and sensitive classification of metagenomic sequences. Genome Res. 26:1721-1729.

Knoth, J. L., Kim, S. H., Ettl, G. J., and Doty, S. L. 2014. Biological nitrogen fixation and biomass accumulation within poplar clones as a result of inoculations with diazotrophic endophyte consortia. New Phytol. 201:599-609.

Kolde, R. 2012. Package 'pheatmap'. Bioconductor 1-6.

Kudla, J., Xu, Q., Harter, K., Gruissem, W., and Luan, S. 1999. Genes for calcineurin B-like proteins in Arabidopsis are differentially regulated by stress signals. Proc. Natl. Acad. Sci. USA 96:4718-4723.

Law, C. W., Chen, Y., Shi, W., and Smyth, G. K. 2014. voom: Precision weights unlock linear model analysis tools for RNA-seq read counts. Genome Biol. 15:R29.

Lin, B., Zhuo, K., Chen, S., Hu, L., Sun, L., Wang, X., et al. 2016. A novel nematode effector suppresses plant immunity by activating host reactive oxygen species-scavenging system. New Phytol. 209:1159-1173.

Liu, L.-L., Ren, H.-M., Chen, L.-Q., Wang, Y., and Wu, W.-H. 2013. A protein kinase, calcineurin B-like protein-interacting protein kinase9, interacts with calcium sensor calcineurin B-like protein 3 and regulates potassium homeostasis under low-potassium stress in Arabidopsis. Plant Physiol. 161:266-277.

Liu, T., Sheng, M., Wang, C. Y., Chen, H., Li, Z., and Tang, M. 2015. Impact of arbuscular mycorrhizal fungi on the growth, water status, and photosynthesis of hybrid poplar under drought stress and recovery. Photosynthetica 53:250-258.

Lohse, M., Nagel, A., Herter, T., May, P., Schroda, M., Zrenner, R., et al. 2014. Mercator: A fast and simple web server for genome scale functional annotation of plant sequence data. Plant Cell Environ. 37:1250-1258.

Ma, Y.-Y., Li, Y.-L., Lai, H.-X., Guo, Q., and Xue, Q.-H. 2017. Effects of two strains of Streptomyces on root-zone microbes and nematodes for biocontrol of root-knot nematode disease in tomato. Appl. Soil Ecol. 112:34-41.

Mandyam, K., Fox, C., and Jumpponen, A. 2012. Septate endophyte colonization and host responses of grasses and forbs native to a tallgrass prairie. Mycorrhiza 22:109-119.

Mandyam, K. G., Roe, J., and Jumpponen, A. 2013. Arabidopsis thaliana model system reveals a continuum of responses to root endophyte colonization. Fungal Biol. 117:250-260.

Marasco, R., Rolli, E., Ettoumi, B., Vigani, G., Mapelli, F., Borin, S., et al. 2012. A drought resistance-promoting microbiome is selected by root system under desert farming. PLoS One 7:e48479.

Markowitz, V. M., Chen, I. M. A., Palaniappan, K., Chu, K., Szeto, E., Grechkin, Y., et al. 2012. IMG: The integrated microbial genomes database and comparative analysis system. Nucleic Acids Res. 40:D115-D122.

Mayerhofer, M. S., Kernaghan, G., and Harper, K. A. 2013. The effects of fungal root endophytes on plant growth: A meta-analysis. Mycorrhiza 23: 119-128.

McDonald, M. C., McDonald, B. A., and Solomon, P. S. 2015. Recent advances in the Zymoseptoria tritici-wheat interaction: Insights from pathogenomics. Front. Plant Sci. 6:1-5.

Meng, S., Zhang, C., Su, L., Li, Y., and Zhao, Z. 2016. Nitrogen uptake and metabolism of Populus simonii in response to PEG-induced drought stress. Environ. Exp. Bot. 123:78-87.

Miller, G., Shulaev, V., and Mittler, R. 2008. Reactive oxygen signaling and abiotic stress. Physiol. Plant. 133:481-489.

Munkvold, L., Kjøller, R., Vestberg, M., Rosendahl, S., and Jakobsen, I 2004. High functional diversity within species of arbuscular mycorrhizal fungi. New Phytol. 164:357-364.

Munsch, P., Geoffroy, V. A., Alatossava, T., and Meyer, J. M. 2000. Application of siderotyping for characterization of Pseudomonas tolaasii and "Pseudomonas reactans" isolates associated with brown blotch disease of cultivated mushrooms. Appl. Environ. Microbiol. 66:4834-4841.

Murata, H., Yamada, A., Maruyama, T., Endo, N., Yamamoto, K., Ohira, T., et al. 2013. Root endophyte interaction between ectomycorrhizal basidiomycete Tricholoma matsutake and arbuscular mycorrhizal tree Cedrela odorata, allowing in vitro synthesis of rhizospheric "shiro". Mycorrhiza 23:235-242.

Nath, M., Bhatt, D., Prasad, R., and Tuteja, N. 2017. Reactive oxygen species (ROS) metabolism and signaling in plant-mycorrhizal association under biotic and abiotic stress conditions. Pages 223-232 in: Mycorrhiza-EcoPhysiology, Secondary Metabolites, Nanomaterials. Springer International.

Naylor, D., Degraaf, S., Purdom, E., and Coleman-Derr, D. 2017. Drought and host selection influence bacterial community dynamics in the grass root microbiome. ISME J. 11:2691-2704.

O'Leary, B., Park, J., Plaxton, W. C., et al. 2011. The remarkable diversity of plant PEPC (phosphoenolpyruvate carboxylase): Recent insights into the physiological functions and post-translational controls of non-photosynthetic PEPCs. Biochem. J. 436:15-34. 
O'Leary, N. A., Wright, M. W., Brister, J. R., Ciufo, S., Haddad, D., and McVeigh, R. 2016. Reference sequence (RefSeq) database at NCBI: Current status, taxonomic expansion, and functional annotation. Nucleic Acids Res.: D733-D745.

Pan, L., Zhang, X., Wang, J., Ma, X., Zhou, M., Huang, L., et al. 2016. Transcriptional profiles of drought-related genes in modulating metabolic processes and antioxidant defenses in Lolium multiflorum. Front. Plant Sci. 7: 519.

Pandey, P., Ramegowda, V., and Senthil-Kumar, M. 2015. Shared and unique responses of plants to multiple individual stresses and stress combinations: physiological and molecular mechanisms. Front. Plant Sci. 6:723.

Petrov, V., Hille, J., Mueller-Roeber, B., and Gechev, T. S. 2015. ROS-mediated abiotic stress-induced programmed cell death in plants. Front. Plant Sci. 6:69.

Piculell, B. J., Hoeksema, J. D., and Thompson, J. N. 2008. Interactions of biotic and abiotic environmental factors in an ectomycorrhizal symbiosis, and the potential for selection mosaics. BMC Biol. 6:23.

Plaxton, W. C. 1996. the organization and regulation of plant glycolysis. Annu. Rev. Plant Physiol. Plant Mol. Biol. 47:185-214.

Qin, S., Feng, W. W., Wang, T. T., Ding, P., Xing, K., and Jiang, J. H. 2017. Plant growth-promoting effect and genomic analysis of the beneficial endophyte Streptomyces sp. KLBMP 5084 isolated from halophyte Limonium sinense. Plant Soil 416:117-132.

Rey, T., and Dumas, B. 2017. Plenty is no plague: Streptomyces symbiosis with crops. Trends Plant Sci. 22:30-37.

Robinson, M. D., and Oshlack, A. 2010. A scaling normalization method for differential expression analysis of RNA-seq data. Genome Biol. 11:R25.

Rodriguez, R. J., Henson, J., Van Volkenburgh, E., Hoy, M., Wright, L., Beckwith, F., et al. 2008. Stress tolerance in plants via habitat-adapted symbiosis. ISME J. 2:404-416.

Rolli, E., Marasco, R., Vigani, G., Ettoumi, B., Mapelli, F., Deangelis, M. L., et al. 2015. Improved plant resistance to drought is promoted by the rootassociated microbiome as a water stress-dependent trait. Environ. Microbiol. 17:316-331

Ruiz-Lozano, J. M., Aroca, R., Zamarreño, Á. M., Molina, S., Andreo-Jiménez, B., Porcel, R., et al. 2016. Arbuscular mycorrhizal symbiosis induces strigolactone biosynthesis under drought and improves drought tolerance in lettuce and tomato. Plant Cell Environ. 39:441-452.

Sah, S. K., Reddy, K. R., and Li, J. 2016. Abscisic acid and abiotic stress tolerance in crop plants. Front. Plant Sci. 7:571.

Santos-Medellín, C., Edwards, J., Liechty, Z., Nguyen, B., and Sundaresan, V. 2017. Drought stress results in a compartment-specific restructuring of the rice root-associated microbiomes. MBio 8:e00764-17.

Sesma, A., and Osbourn, A. E. 2004. The rice leaf blast pathogen undergoes developmental processes typical of root-infecting fungi. Nature 431:582-586.
Shahid, S. A., and Al-Shankiti, A. 2013. Sustainable food production in marginal lands-Case of GDLA member countries. Int. Soil Water Conserv. Res. 1: 24-38.

Sinha, R., Gupta, A., and Senthil-Kumar, M. 2016. Understanding the impact of drought on foliar and xylem invading bacterial pathogen stress in chickpea. Front. Plant Sci. 7:808.

Sousa, J. A. de J., and Olivares, F. L. 2016. Plant growth promotion by Streptomycetes: Ecophysiology, mechanisms and applications. Chem. Biol. Technol. Agric. 3:24

Tang, S., Dong, Y., Liang, D., Zhang, Z., Ye, C. Y., Shuai, P., et al. 2015. Analysis of the drought stress-responsive transcriptome of black cottonwood (Populus trichocarpa) using Deep RNA sequencing. Plant Mol. Biol. Rep. 33:424-438.

Thimm, O., Bläsing, O., Gibon, Y., Nagel, A., Meyer, S., Krüger, P., et al. 2004. MAPMAN: A user-driven tool to display genomics data sets onto diagrams of metabolic pathways and other biological processes. Plant J. 37:914-939.

Timm, C. M., Carter, K. R., Carrell, A. A., Jun, S., Jawdy, S. S., Vélez, J. M., et al. 2018. Abiotic stresses shift belowground Populus-associated bacteria toward a core stress microbiome. mSystems 3:e00070-17.

Truong, D. T., Franzosa, E. A., Tickle, T. L., Scholz, M., Weingart, G., Shuai, P., et al. 2015. MetaPhlAn2 for enhanced metagenomic taxonomic profiling. Nat. Methods 12:902-903.

Tuteja, N. 2007. Abscisic acid and abiotic stress signaling. Plant Signal. Behav. 2:135-138

Viger, M., Smith, H. K., Cohen, D., Dewoody, J., Trewin, H., Steenackers, M., et al. 2016. Adaptive mechanisms and genomic plasticity for drought tolerance identified in European black poplar (Populus nigra L.). Tree Physiol. 36: 909-928.

Vurukonda, S. S. K. P., Vardharajula, S., Shrivastava, M., and SkZ, A. 2016. Enhancement of drought stress tolerance in crops by plant growth promoting rhizobacteria. Microbiol. Res. 184:13-24.

Wood, D. E., and Salzberg, S. L. 2014. Kraken: Ultrafast metagenomic sequence classification using exact alignments. Genome Biol. 15:R46.

Yandigeri, M. S., Meena, K. K., Singh, D., Malviya, N., Singh, D. P., Solanki, M. K., et al. 2012. Drought-tolerant endophytic actinobacteria promote growth of wheat (Triticum aestivum) under water stress conditions. Plant Growth Regul. 68:411-420.

Zandalinas, S. I., Mittler, R., Balfagón, D., Arbona, V., and Gómez-Cadenas, A. 2017. Plant adaptations to the combination of drought and high temperatures. Physiol. Plant. 162:2-12.

Zhang, H., Wei, S., Hu, W., Xiao, L., and Tang, M. 2017. Arbuscular mycorrhizal fungus Rhizophagus irregularis increased potassium content and expression of genes encoding potassium channels in Lycium barbarum. Front. Plant Sci. 8. 